PROCEEDINGS OF THE

AMERICAN MATHEMATICAL SOCIETY

Volume 125, Number 8, August 1997, Pages 2255-2263

S 0002-9939(97)03874-4

\title{
ON THE RATIONAL CUSPIDAL SUBGROUP AND THE RATIONAL TORSION POINTS OF $J_{0}(p q)$
}

\author{
SENG-KIAT CHUA AND SAN LING \\ (Communicated by William W. Adams)
}

\begin{abstract}
For two distinct prime numbers $p$, $q$, we compute the rational cuspidal subgroup $C(p q)$ of $J_{0}(p q)$ and determine the $\ell$-primary part of the rational torsion subgroup of the old subvariety of $J_{0}(p q)$ for most primes $\ell$. Some results of Berkovič on the nontriviality of the Mordell-Weil group of some Eisenstein factors of $J_{0}(p q)$ are also refined.
\end{abstract}

\section{INTRODUCTION}

Let $p, q$ be two distinct prime numbers, and let $X_{0}(p), X_{0}(q)$ and $X_{0}(p q)$ denote the classical modular curves over $\mathbb{Q}$. Let $J_{0}(p), J_{0}(q)$ and $J_{0}(p q)$ denote the corresponding Jacobian varieties, also defined over $\mathbb{Q}$.

On $X_{0}(p)$ (resp. $X_{0}(q)$ ), there are two (rational) cusps $P_{1}$ and $P_{p}$ (resp. $P_{1}$ and $P_{q}$ ). It is well-known that the cuspidal subgroup $C(p)$ (resp. $C(q)$ ), generated by the class of the cuspidal divisor $P_{1}-P_{p}$ (resp. $\left.P_{1}-P_{q}\right)$, is cyclic of order $\frac{p-1}{(p-1,12)}$ (resp. $\left.\frac{q-1}{(q-1,12)}\right)$ (cf. [7]).

There are exactly four cusps, denoted $P_{1}, P_{p}, P_{q}$ and $P_{p q}$, on $X_{0}(p q)$, and they are all rational. Let $C_{p}=P_{1}-P_{p}, C_{q}=P_{1}-P_{q}$ and $C_{p q}=P_{1}-P_{p q}$ be the cuspidal divisors of degree 0 . For a degree- 0 divisor $D$ on $X_{0}(p q)$, we shall denote the divisor class it defines by $\bar{D}$.

The cuspidal subgroup $C(p q)$ of $J_{0}(p q)$ is clearly generated by $\bar{C}_{p}, \bar{C}_{q}$ and $\bar{C}_{p q}$. Manin [5] has shown that $\bar{C}_{p}, \bar{C}_{q}$ and $\bar{C}_{p q}$ are of finite order. For each prime $\ell$, we shall let $r_{\ell}, s_{\ell}$ and $t_{\ell}$ be the non-negative integers such that $\ell^{r_{\ell}}\left(\right.$ resp. $\left.\ell^{s_{\ell}}, \ell^{t_{\ell}}\right)$ is the exact power of $\ell$ dividing the order of $\bar{C}_{p}$ (resp. $\bar{C}_{q}, \bar{C}_{p q}$ ). For an integer $x$, let $v_{\ell}(x)$ be the valuation of $x$ at $\ell$, i.e., $\ell^{v_{\ell}(x)}$ is the exact power of $\ell$ dividing $x$. Then we also set, for each prime $\ell$,

$$
\begin{aligned}
& m_{\ell} \stackrel{\text { def }}{=} \min \left(v_{\ell}\left(\frac{p-1}{(p-1,12)}\right), v_{\ell}\left(\frac{q-1}{(q-1,12)}\right)\right), \\
& M_{\ell} \stackrel{\text { def }}{=} \max \left(v_{\ell}(p-1), v_{\ell}(q-1)\right) .
\end{aligned}
$$

In this article, we compute the rational cuspidal subgroup $C(p q)$ of $J_{0}(p q)$. Here, for a finite group $G$, the notation $G_{\ell}$ represents the $\ell$-primary part of $G$.

Received by the editors September 8, 1995 and, in revised form, March 10, 1996.

1991 Mathematics Subject Classification. Primary 11G18, 11F03, 11F20, 14H40.

The authors would like to thanks Ken Ribet for private communication. We are also grateful to the referee for comments which helped improve the presentation of the paper.

(C)1997 American Mathematical Society 
Theorem 1. (i) Let $p, q \geq 5$ be two distinct primes. For any prime $\ell$, the $\ell$-primary part of the cuspidal subgroup $C(p q)$ of $J_{0}(p q)$ is given by:

$$
\begin{array}{ll}
C(p q)_{\ell} \cong \mathbb{Z} / \ell^{r_{\ell}} \mathbb{Z} \times \mathbb{Z} / \ell^{s_{\ell}} \mathbb{Z} & \text { if } \quad \ell \times\left(\frac{p-1}{2}, \frac{q-1}{2}\right), \\
C(p q)_{\ell} \cong \mathbb{Z} / \ell^{r_{\ell}} \mathbb{Z} \times \mathbb{Z} / \ell^{M_{\ell}} \mathbb{Z} \times \mathbb{Z} / \ell^{m_{\ell}} \mathbb{Z} & \text { if } \quad \ell \mid\left(\frac{p-1}{2}, \frac{q-1}{2}\right), \quad \ell \geq 5, \\
C(p q)_{\ell} \cong \mathbb{Z} / \ell^{r_{\ell}} \mathbb{Z} \times \mathbb{Z} / \ell^{M_{\ell}-1} \mathbb{Z} \times \mathbb{Z} / \ell^{m_{\ell}} \mathbb{Z} & \text { if } \quad \ell \mid\left(\frac{p-1}{2}, \frac{q-1}{2}\right), \quad \ell=2,3 .
\end{array}
$$

(ii) For $p \geq 5$, the cuspidal subgroup $C(3 p)$ of $J_{0}(3 p)$ is given by:

$$
C(3 p) \cong\left\{\begin{array}{llll}
\mathbb{Z} / \frac{p-1}{3} \mathbb{Z} \times \mathbb{Z} / \frac{p^{2}-1}{12} \mathbb{Z} & \text { if } & p \equiv 1 \quad(\bmod 3), \\
\mathbb{Z} /(p-1) \mathbb{Z} \times \mathbb{Z} / \frac{p^{2}-1}{12} \mathbb{Z} & \text { if } & p \equiv 2 \quad(\bmod 3) .
\end{array}\right.
$$

(iii) For $p \geq 5$, the cuspidal subgroup $C(2 p)$ of $J_{0}(2 p)$ is given by:

$$
C(2 p) \cong\left\{\begin{array}{llll}
\mathbb{Z} / \frac{p-1}{(p-1,8)} \mathbb{Z} \times \mathbb{Z} / \frac{p^{2}-1}{24} \mathbb{Z} & \text { if } p \neq 1 \quad(\bmod 8), \\
\mathbb{Z} / \frac{p-1}{4} \mathbb{Z} \times \mathbb{Z} / \frac{p^{2}-1}{24} \mathbb{Z} & \text { if } p \equiv 1 \quad(\bmod 8) .
\end{array}\right.
$$

and $C(6)=0$.

After some general discussion on the generators of $C(p q)$ and establishing some relations among these generators, we prove Theorem 1 (i) in Section 3.3. The proofs of Theorem 1 (ii) and (iii) are left to the reader.

Recall that there are degeneracy maps $v_{1}, v_{q}: X_{0}(p q) \rightarrow X_{0}(p)$ and $v_{1}, v_{p}$ : $X_{0}(p q) \rightarrow X_{0}(q)$. They induce via Pic functoriality the maps $v_{1}^{*}, v_{q}^{*}: J_{0}(p) \rightarrow$ $J_{0}(p q)$ and $v_{1}^{*}, v_{p}^{*}: J_{0}(q) \rightarrow J_{0}(p q)$, and via Albanese functoriality the maps $\left(v_{1}\right)_{*},\left(v_{q}\right)_{*}: J_{0}(p q) \rightarrow J_{0}(p)$ and $\left(v_{1}\right)_{*},\left(v_{p}\right)_{*}: J_{0}(p q) \rightarrow J_{0}(q)$. Let $\gamma$ be the map

$$
\gamma=\left(v_{1}^{*} \times v_{q}^{*}\right) \times\left(v_{1}^{*} \times v_{p}^{*}\right): J_{0}(p)^{2} \times J_{0}(q)^{2} \rightarrow J_{0}(p q) .
$$

The image of $\gamma$ is the old subvariety of $J_{0}(p q)$, which we denote by $J_{\text {old }}$. The group of $\mathbb{Q}$-rational torsion points of this old subvariety is denoted by $J_{\text {old }}(\mathbb{Q})_{\text {tor }}$. We prove in Section 4

Theorem 2. Let $\ell$ be an odd prime not dividing $\left(\frac{p-1}{(p-1,24)}, \frac{q-1}{(q-1,24)}\right)$; then the restriction of $\gamma$ to $C(p)_{\ell}^{2} \times C(q)_{\ell}^{2}$ defines an isomorphism

$$
C(p)_{\ell}^{2} \times C(q)_{\ell}^{2} \cong\left(J_{\text {old }}(\mathbb{Q})_{\text {tor }}\right)_{\ell}
$$

Theorems 1 and 2 can be used to refine some results of Berkovič [1] on the nontriviality of the Mordell-Weil group of some Eisenstein factors of $J_{0}(p q)$.

\section{The Dedekind $\eta$-Functions And Cuspidal Divisors}

2.1. The Dedekind $\eta$-functions. Let $N$ be a positive integer, and let $\delta$ denote a positive divisor of $N$. Let $\mathbf{r}=\left(r_{\delta}\right)$ be a family of rational numbers $r_{\delta} \in \mathbb{Q}$ indexed by all the positive divisors $\delta$ of $N$. Let

$$
g_{\mathbf{r}}=\prod_{\delta \mid N} \eta_{\delta}^{r_{\delta}}
$$

be made up from the Dedekind $\eta$-functions, where $\eta_{\delta}(z) \stackrel{\text { def }}{=} \eta(\delta z)$. We recall without proof the following proposition (cf. [2, Proposition 3.2.1] or [3, Proposition 1]): 
Proposition 1. The function $g_{\mathbf{r}}$ in (2) is a modular function on the modular curve $X_{0}(N)$ if and only if the following conditions are satisfied:

(0) all the $r_{\delta}$ are rational integers;

(1) $\sum_{\delta \mid N} r_{\delta} \delta \equiv 0(\bmod 24)$;

(2) $\sum_{\delta \mid N} r_{\delta} \frac{N}{\delta} \equiv 0(\bmod 24)$;

(3) $\sum_{\delta \mid N} r_{\delta}=0$;

(4) $\prod_{\delta \mid N} \delta^{r_{\delta}}$ is the square of a rational number.

By applying [4, Proposition 2] to the case $N=p q$, we have a bijection $\Lambda$ from $\left\{\prod_{\delta \mid p q} \eta_{\delta}^{r_{\delta}}: r_{\delta} \in \mathbb{Q}\right.$ and $\left.\sum_{\delta \mid p q} r_{\delta}=0\right\}$ to $\left\{\sum_{d \mid p q} m_{d} P_{d}: m_{d} \in \mathbb{Q}\right.$ and $\left.\sum_{d \mid p q} m_{d}=0\right\}$. Identifying the first set with

$$
\left\{\left(\begin{array}{l}
r_{1} \\
r_{p} \\
r_{q} \\
r_{p q}
\end{array}\right) \in \mathbb{Q}^{4}: r_{1}+r_{p}+r_{q}+r_{p q}=0\right\}
$$

and identifying the second set with

$$
\left\{\left(\begin{array}{l}
m_{1} \\
m_{p} \\
m_{q} \\
m_{p q}
\end{array}\right) \in \mathbb{Q}^{4}: m_{1}+m_{p}+m_{q}+m_{p q}=0\right\}
$$

the map $\Lambda$ may be represented as a matrix

$$
\Lambda=\frac{1}{24}\left(\begin{array}{rrrr}
p q & q & p & 1 \\
q & p q & 1 & p \\
p & 1 & p q & q \\
1 & p & q & p q
\end{array}\right)
$$

so

$$
\Lambda^{-1}=\frac{24}{\left(p^{2}-1\right)\left(q^{2}-1\right)}\left(\begin{array}{rrrr}
p q & -q & -p & 1 \\
-q & p q & 1 & -p \\
-p & 1 & p q & -q \\
1 & -p & -q & p q
\end{array}\right)
$$


2.2. Orders of cuspidal divisors. Using the identification above, we have

$$
\begin{aligned}
C_{p} & =\left(\begin{array}{r}
1 \\
-1 \\
0 \\
0
\end{array}\right) \quad \text { and } \quad \Lambda^{-1} C_{p}=\frac{24}{(p-1)\left(q^{2}-1\right)}\left(\begin{array}{r}
q \\
-q \\
-1 \\
1
\end{array}\right) ; \\
C_{q} & =\left(\begin{array}{r}
1 \\
0 \\
-1 \\
0
\end{array}\right) \quad \text { and } \Lambda^{-1} C_{q}=\frac{24}{\left(p^{2}-1\right)(q-1)}\left(\begin{array}{r}
p \\
-1 \\
-p \\
1
\end{array}\right) ; \\
C_{p q} & =\left(\begin{array}{r}
1 \\
0 \\
0 \\
-1
\end{array}\right) \quad \text { and } \quad \Lambda^{-1} C_{p q}=\frac{24}{\left(p^{2}-1\right)\left(q^{2}-1\right)}\left(\begin{array}{c}
p q-1 \\
p-q \\
q-p \\
1-p q
\end{array}\right) .
\end{aligned}
$$

Proposition 1 can then be used to determine the orders of the divisor classes $\bar{C}_{p}, \bar{C}_{q}$ and $\bar{C}_{p q}$. We summarize the results of our computations as follows:

\begin{tabular}{|c|cc|c|cc|}
\hline & \multicolumn{3}{|c|}{ order of $\bar{C}_{p}$} & order of $\bar{C}_{q}$ & \multicolumn{3}{c|}{ order of $\bar{C}_{p q}$} \\
\hline$q=2$ & $\frac{p-1}{(p-1,8)}$ & if $p \not \equiv 1(\bmod 8)$ & $\frac{p^{2}-1}{24}$ & $\frac{p^{2}-1}{8}$ & if $p \equiv 1(\bmod 3)$ \\
$p \geq 5$ & $\frac{p-1}{4}$ & if $p \equiv 1(\bmod 8)$ & $\frac{p^{2}-1}{24}$ & if $p \equiv 2(\bmod 3)$ \\
\hline$q=3$ & $\frac{p-1}{3}$ & if $p \equiv 1(\bmod 3)$ & $\frac{p^{2}-1}{12}$ & $\frac{p^{2}-1}{12}$ & if $p \equiv 3(\bmod 4)$ \\
$p \geq 5$ & $p-1$ & if $p \equiv 2(\bmod 3)$ & $\frac{p^{2}-1}{6}$ & if $p \equiv 1(\bmod 4)$ \\
\hline$q \geq 5$ & & $\frac{\left(q^{2}-1\right)(p-1)}{24}$ & $\frac{\left(p^{2}-1\right)(q-1)}{24}$ & & $\frac{\left(p^{2}-1\right)\left(q^{2}-1\right)}{m}$ \\
$p \geq 5$ & & & & \\
\hline
\end{tabular}

where $m \stackrel{\text { def }}{=}\left(\left(p^{2}-1\right)\left(q^{2}-1\right), 24(p q-1), 24(p-q), 12(p-1)(q+1), 12(q-1)(p+1)\right)$.

\section{Relations among the generators $\bar{C}_{p}, \bar{C}_{q}, \bar{C}_{p q}$}

3.1. The general strategy. To establish relations among the generators $\bar{C}_{i}$ of $C(p q)$ we first apply the maps $v_{1}^{*}, v_{q}^{*}: J_{0}(p) \rightarrow J_{0}(p q)$ and $v_{1}^{*}, v_{p}^{*}: J_{0}(q) \rightarrow J_{0}(p q)$ to translate any known relations in $J_{0}(p)$ and $J_{0}(q)$ to some relations in $J_{0}(p q)$. Next, we note that a relation $\sum \lambda_{i} \bar{C}_{i}=0$ exists if and only if $\Lambda^{-1}\left(\sum \lambda_{i} C_{i}\right)$ satisfies the conditions of Proposition 1. Using this criterion, one can easily check the validity of any relation. In addition, we can simplify the checking process by applying the maps $\left(v_{1}\right)_{*},\left(v_{q}\right)_{*}: J_{0}(p q) \rightarrow J_{0}(p)\left(\right.$ resp. $\left.\left(v_{1}\right)_{*},\left(v_{p}\right)_{*}: J_{0}(p q) \rightarrow J_{0}(q)\right)$ to translate a relation in $J_{0}(p q)$ to a relation in $J_{0}(p)$ (resp. $J_{0}(q)$ ).

In this section, we will apply this strategy to establish the relations among the generators $\bar{C}_{p}, \bar{C}_{q}, \bar{C}_{p q}$. Note that a similar strategy has been used in [4, Section 2] to establish the relations among the generators of $C\left(p^{r}\right)$, where $p \geq 5$ is a prime. Consequently, we will only state the key lemmas in Section 3.2 since most of the details are similar to those in [4, Section 2].

3.2. The case $p, q \geq 5$. We now determine the relations involving the generators $\bar{C}_{p}, \bar{C}_{q}$ and $\bar{C}_{p q}$ of $C(p q)$ by finding such relations at each prime $\ell$. For each prime $\ell$, we may write

$$
\frac{\left(q^{2}-1\right)(p-1)}{24}=\ell^{r_{\ell}} \ell_{1}, \quad \frac{\left(p^{2}-1\right)(q-1)}{24}=\ell^{s_{\ell}} \ell_{2}, \quad \frac{\left(q^{2}-1\right)\left(p^{2}-1\right)}{m}=\ell^{t_{\ell}} \ell_{3}
$$


where $\ell \backslash \ell_{1}, \ell \chi \ell_{2}, \ell \backslash \ell_{3}$. We also set

$$
C_{p, \ell}=\ell_{1} \ell_{2} \ell_{3} C_{p}, \quad C_{q, \ell}=\ell_{1} \ell_{2} \ell_{3} C_{q}, \quad C_{p q, \ell}=\ell_{1} \ell_{2} \ell_{3} C_{p q} .
$$

Since $\left(\ell_{1} \ell_{2}, \ell\right)=\left(\ell_{2} \ell_{3}, \ell\right)=\left(\ell_{1} \ell_{3}, \ell\right)=1$, it is clear that $\bar{C}_{p, \ell}, \bar{C}_{q, \ell}$ and $\bar{C}_{p q, \ell}$ generate $C(p q)_{\ell}$. By applying the maps $v_{1}^{*}, v_{q}^{*}: J_{0}(p) \rightarrow J_{0}(p q)$ and $v_{1}^{*}, v_{p}^{*}: J_{0}(q) \rightarrow J_{0}(p q)$ as described in Section 3.1, we get

Lemma 1. (i) If $\ell$ does not divide $\left(\frac{q-1}{(q-1,12)}, \frac{p-1}{(p-1,12)}\right)$, then $C(p q)_{\ell}$ is generated by $\bar{C}_{p, \ell}$ and $\bar{C}_{q, \ell}$.

(ii) If $\ell$ divides $\left(\frac{q-1}{(q-1,12)}, \frac{p-1}{(p-1,12)}\right)$, then $\ell^{m_{\ell}} \bar{C}_{p q, \ell}=\alpha \bar{C}_{p, \ell}+\beta \bar{C}_{q, \ell}$ for some $\alpha, \beta$.

Furthermore, by applying the maps $\left(v_{1}\right)_{*},\left(v_{q}\right)_{*}: J_{0}(p q) \rightarrow J_{0}(p)$ and $\left(v_{1}\right)_{*},\left(v_{p}\right)_{*}$ : $J_{0}(p q) \rightarrow J_{0}(q)$ together with the criterion stated in Section 3.1, we have

Lemma 2. There is no non-trivial relation

$$
\nu_{\ell} \bar{C}_{p q, \ell}=\lambda_{\ell} \bar{C}_{p, \ell}+\mu_{\ell} \bar{C}_{q, \ell}
$$

with $0 \leq \lambda_{\ell}<\ell^{r_{\ell}}, 0 \leq \mu_{\ell}<\ell^{s_{\ell}}$ and $0 \leq \nu_{\ell}<\ell^{m_{\ell}}$.

To help the reader in reconstructing the proof, we include the following formulas:

$$
\begin{aligned}
v_{1}^{*}\left(C_{p}\right) & =-C_{q}+q C_{p}+C_{p q}, \\
v_{q}^{*}\left(C_{p}\right) & =-q C_{q}+C_{p}+q C_{p q}, \\
\left(v_{1}\right)_{*}\left(C_{p}\right) & =C_{p}, \\
\left(v_{1}\right)_{*}\left(C_{q}\right) & =0, \\
\left(v_{1}\right)_{*}\left(C_{p q}\right) & =C_{p} .
\end{aligned}
$$

In addition, the two maps $v_{1}$ and $v_{q}$ are related by the formula $v_{1}=v_{q} \circ w_{q}$ where $w_{q}$ is an Atkin-Lehner involution (see the remark after Theorem 3).

From Lemma 1 (ii) and Lemma 2, if there is any relation

$$
\nu_{\ell} \bar{C}_{p q, \ell}=\lambda_{\ell} \bar{C}_{p, \ell}+\mu_{\ell} \bar{C}_{q, \ell}
$$

relating $\bar{C}_{p q, \ell}, \bar{C}_{p, \ell}$ and $\bar{C}_{q, \ell}$, we may assume $v_{\ell}\left(\nu_{\ell}\right) \geq m_{\ell}$. Together with Lemma 1 (ii), this relation can be reduced to

$$
0=\lambda_{\ell} \bar{C}_{p, \ell}+\mu_{\ell} \bar{C}_{q, \ell} .
$$

Therefore, we shall next study relations of this type.

Lemma 3. Suppose (5) is satisfied.

(i) If $\ell \times(p-1, q-1)($ hence $\ell \neq 2)$, then $\lambda_{\ell}=\mu_{\ell}=0$.

(ii) If $\ell \mid(p-1, q-1)$ and $\ell \neq 2$, then $\lambda_{\ell}$ and $\mu_{\ell}$ satisfy

$$
\begin{aligned}
& \lambda_{\ell} \ell_{2} q \equiv-\mu_{\ell} \ell_{1} \equiv \lambda_{\ell} \ell_{2} \quad\left(\bmod \ell^{r_{\ell}}\right) \quad(\ell \geq 3) \\
& \text { and } \lambda_{\ell} \equiv \mu_{\ell} \equiv 0 \quad\left(\bmod \ell^{M_{\ell}}\right) \quad(\ell \geq 5) \quad \text { or } \quad \lambda_{3} \equiv \mu_{3} \equiv 0 \quad\left(\bmod 3^{M_{3}-1}\right) .
\end{aligned}
$$

(iii) If at least one of $p, q \equiv 3(\bmod 4)$, then $\lambda_{2}=\mu_{2}=0$.

(iv) If $p \equiv q \equiv 1(\bmod 4)$, then $\lambda_{2}$ and $\mu_{2}$ satisfy

$$
\lambda_{2} \ell_{2} q \equiv-\mu_{2} \ell_{1} \equiv \lambda_{2} \ell_{2} \quad\left(\bmod 2^{r_{2}}\right) \quad \text { and } \quad \lambda_{2} \equiv \mu_{2} \equiv 0 \quad\left(\bmod 2^{M_{2}-1}\right) .
$$


3.3. Proof of Theorem 1 (i). To prove Theorem 1 (i), note that if $\ell$ does not divide $\left(\frac{p-1}{2}, \frac{q-1}{2}\right)$, then $\ell$ does not divide $\left(\frac{p-1}{(p-1,12)}, \frac{q-1}{(q-1,12)}\right)$. Therefore $C(p q)_{\ell}$ is generated by $\bar{C}_{p, \ell}$ and $\bar{C}_{q, \ell}$. By Lemma 3 (i) and (iii), there is no relation between $\bar{C}_{p, \ell}$ and $\bar{C}_{q, \ell}$ in this case. The orders of $\bar{C}_{p, \ell}$ and $\bar{C}_{q, \ell}$ are given by (3). In the remaining case, Lemma 1 (ii) and Lemma 2 show that

$$
C(p q)_{\ell} \cong\left\langle\bar{C}_{p, \ell}, \bar{C}_{q, \ell}\right\rangle \times \mathbb{Z} / \ell^{m_{\ell}} \mathbb{Z} .
$$

If $\ell$ divides $\left(\frac{p-1}{2}, \frac{q-1}{2}\right)$, then $\ell$ divides $(p-1, q-1)$.

If $\ell \geq 5$, Lemma 3 (ii) shows that $\left\langle\bar{C}_{p, \ell}, \bar{C}_{q, \ell}\right\rangle \cong \mathbb{Z} / \ell^{r_{\ell}} \mathbb{Z} \times \mathbb{Z} / \ell^{M_{\ell}} \mathbb{Z}$.

If $\ell=3$, Lemma 3 (ii) shows that $\left\langle\bar{C}_{p, 3}, \bar{C}_{q, 3}\right\rangle \cong \mathbb{Z} / 3^{r_{3}} \mathbb{Z} \times \mathbb{Z} / 3^{M_{3}-1} \mathbb{Z}$.

If $\ell=2$, Lemma 3 (iv) shows that $\left\langle\bar{C}_{p, 2}, \bar{C}_{q, 2}\right\rangle \cong \mathbb{Z} / 2^{r_{2}} \mathbb{Z} \times \mathbb{Z} / 2^{M_{2}-1} \mathbb{Z}$.

This completes the proof of Theorem 1 (i).

3.4. Additional remarks on Theorem 1. Consider the following cuspidal divisors (cf. [8]):

\begin{tabular}{|l|c|}
\hline divisor & order of divisor class \\
\hline$D^{+,-}=P_{1}+P_{p}-P_{q}-P_{p q}$ & $\frac{(p+1)(q-1)}{((p+1)(q-1), 24)}$ \\
\hline$D^{-,+}=P_{1}-P_{p}+P_{q}-P_{p q}$ & $\frac{(p-1)(q+1)}{((p-1)(q+1), 24)}$ \\
\hline$D^{-,-}=P_{1}-P_{p}-P_{q}+P_{p q}$ & $\frac{(p-1)(q-1)}{((p-1)(q-1), 24)}$ \\
\hline
\end{tabular}

Note that we have the inclusions of groups

$$
\left\langle 2 \bar{C}_{p}, 2 \bar{C}_{q}, 2 \bar{C}_{p q}\right\rangle \subseteq\left\langle\overline{D^{+,-}}, \overline{D^{-,+}}, \overline{D^{-,-}}\right\rangle \subseteq C(p q)=\left\langle\bar{C}_{p}, \bar{C}_{q}, \bar{C}_{p q}\right\rangle
$$

If we are only interested in the odd part of $C(p q)$, we may as well consider the odd part of $\left\langle\overline{D^{+,-}}, \overline{D^{-,+}}, \overline{D^{-,-}}\right\rangle$. Using techniques employed in the earlier sections, we can show that there are no non-trivial relations among $\overline{D^{+,-}}, \overline{D^{-,+}}$and $\overline{D^{-,-}}$(even at the 2-primary part). Therefore the odd part of $C(p q)$ is isomorphic to that of $\left\langle\overline{D^{+,-}}\right\rangle \times\left\langle\overline{D^{-,+}}\right\rangle \times\left\langle\overline{D^{-,-}}\right\rangle$.

\section{RATIONAL TORSION POINTS OF THE OLD SUBVARIETY}

The goal of this section is to establish Theorem 2. Let $\gamma$ be the map in (1), let $K$ denote the kernel of $\gamma$ and let $\ell$ be an odd prime. Let $\Sigma(p)($ resp. $\Sigma(q))$ denote the Shimura subgroup of $J_{0}(p)$ (resp. $\left.J_{0}(q)\right)$; it is a cyclic group of order $\frac{p-1}{(p-1,12)}$ (resp. $\left.\frac{q-1}{(q-1,12)}\right)$. Let $\overline{C(p)}$ be the image of the anti-diagonal $C(p)$ in $J_{0}(p q)$, i.e.,

$$
\overline{C(p)}=\left\{x \in J_{0}(p q): x=\left(v_{1}^{*}-v_{q}^{*}\right)(c) \text { for } c \in C(p)\right\},
$$

and $\overline{C(q)}$ is similarly defined. It is shown in [9] that

$$
K_{\ell} \cong \Sigma(p)_{\ell} \times \Sigma(q)_{\ell} \times\left(\overline{C(p)}_{\ell} \cap \overline{C(q)}_{\ell}\right) .
$$

Moreover, it is known that the group $\overline{C(p)} \cap \overline{C(q)}$ has order $\left(\frac{p-1}{(p-1,24)}, \frac{q-1}{(q-1,24)}\right)$.

Let $P \subseteq J_{0}(p)^{2} \times J_{0}(q)^{2}$ be defined as $P=\gamma^{-1}\left(J_{\text {old }}(\mathbb{Q})_{\text {tor }}\right)$. For each odd prime $\ell$, we have an exact sequence of $\operatorname{Gal}(\overline{\mathbb{Q}} / \mathbb{Q})$-modules

$$
0 \longrightarrow K_{\ell} \longrightarrow P_{\ell} \stackrel{\gamma}{\longrightarrow}\left(J_{\text {old }}(\mathbb{Q})_{\text {tor }}\right)_{\ell} \longrightarrow 0 .
$$


Now let $r$ be a prime distinct from $p$ and $q$. Let $T_{r}$ be the $r$ th Hecke operator on $J_{0}(p q)$. Since $(r, p q)=1, T_{r}$ acts on $J_{0}(p)^{2} \times J_{0}(q)^{2}$ (hence on $P$ ) diagonally, i.e., it acts on $J_{0}(p)$ (resp. $\left.J_{0}(q)\right)$ like the usual Hecke operator $T_{r}$ in $\mathbb{T}_{p}=\operatorname{End}_{\mathbb{Q}}\left(J_{0}(p)\right)$ (resp. $\mathbb{T}_{q}=\operatorname{End}_{\mathbb{Q}}\left(J_{0}(q)\right)$ ) (cf. [9], "Formulaire").

Since $\Sigma(p)$ and $C(p)$ (resp. $\Sigma(q)$ and $C(q)$ ) are Eisenstein, i.e., they are annihilated by $T_{r}-(1+r)$ for all primes $r \neq p$ (resp. $r \neq q$ ), it follows from the isomorphism (6) that $\left(T_{r}-(1+r)\right) K_{\ell}=0$ for all primes $r \neq p, q$. By considering the action of $T_{r}$ on the Néron model of $J_{0}(p q)$ over $\mathbb{Z}$ and restricting to characteristic $r$, the Eichler-Shimura relation yields the equation

$$
T_{r}=\text { Frob }_{r}+r / \text { Frob }_{r}
$$

on $J_{0}(p q) / \mathbb{F}_{r}$ where Frob $_{r}$ denotes the Frobenius at $r$. This implies, in particular, $\left(T_{r}-(1+r)\right)\left(J_{\text {old }}(\mathbb{Q})_{\text {tor }}\right)_{\ell}=0$. Since $T_{r}-(1+r)$ kills $K_{\ell}$ and $\left(J_{\text {old }}(\mathbb{Q})_{\text {tor }}\right)_{\ell}$, and $T_{r}-(1+r)$ commutes with the action of $\operatorname{Gal}(\overline{\mathbb{Q}} / \mathbb{Q}), T_{r}-(1+r)$ induces (using the Snake Lemma) a homomorphism $\delta_{r}:\left(J_{\text {old }}(\mathbb{Q})_{\text {tor }}\right)_{\ell} \rightarrow K_{\ell}^{\mathrm{Gal}(\overline{\mathbb{Q}} / \mathbb{Q})}$. When $\ell$ does not divide $\left(\frac{p-1}{(p-1,24)}, \frac{q-1}{(q-1,24)}\right), \overline{C(p)}_{\ell} \cap \overline{C(q)}_{\ell}$ is trivial, and (6) implies that $K_{\ell}^{\mathrm{Gal}(\overline{\mathbb{Q}} / \mathbb{Q})}$ is trivial. Therefore $\delta_{r}$ is the trivial homomorphism. This means that $T_{r}-(1+r)$ annihilates $P_{\ell}$, for all primes $r \neq p, q$.

Lemma 4 (cf. [11]). Let $S$ be a set of primes of density one that does not contain $p$. The ideal in $\mathbb{T}_{p}=\operatorname{End}_{\mathbb{Q}}(J(p))$ generated by $\left\{T_{r}-(1+r): r \in S\right\}$ is the Eisenstein ideal $I_{p}$.

Proof. Let $I$ denote the ideal in $\mathbb{T}_{p}$ generated by $\left\{T_{r}-(1+r): r \in S\right\}$ and let $\mathfrak{m}$ denote a maximal ideal of $\mathbb{T}_{p}$ containing $\left\{T_{r}-(1+r): r \in S\right\}$. By [10, Theorem 5.2], $\mathfrak{m}$ gives rise to a reducible Galois representation $\rho_{\mathfrak{m}}: \operatorname{Gal}(\overline{\mathbb{Q}} / \mathbb{Q}) \rightarrow \operatorname{GL}\left(2, \mathbb{T}_{p} / \mathfrak{m}\right)$ satisfying

$$
\operatorname{Tr}\left(\rho_{\mathfrak{m}}\left(\operatorname{Frob}_{r}\right)\right) \equiv T_{r} \quad(\bmod \mathfrak{m}), \quad \operatorname{det}\left(\rho_{\mathfrak{m}}\left(\operatorname{Frob}_{r}\right)\right) \equiv r \quad(\bmod \mathfrak{m})
$$

for almost all $r$. Proposition 14.1 of $[6$, Chapter II] shows that $\mathfrak{m}$ is Eisenstein. Hence, if $\mathfrak{m} \subset \mathbb{T}_{p}$ is a maximal ideal, $\mathfrak{m} \supset\left\{T_{r}-(1+r): r \in S\right\}$ if and only if $\mathfrak{m} \supset I_{p}$.

To show that $I=I_{p}$, it suffices to demonstrate that $I_{\mathfrak{m}}=I_{p, \mathfrak{m}}$ (in $\mathbb{T}_{\mathfrak{m}}$ ) for all Eisenstein primes $\mathfrak{m}$. By [6, Chapter II, Proposition 16.6], the ideal $I_{p, \mathfrak{m}}$ is a principal ideal in $\mathbb{T}_{p, \mathfrak{m}}$, generated by $T_{r}-(1+r)$ for any "good" prime $r$. (For the definition of a "good" prime, see [6, Chapter II, Section 16].) The set of "good" primes has Dirichlet density $\frac{r-2}{r-1}$ if $r>2$, and $\frac{1}{4}$ if $r=2$. In particular, there is a good prime $r_{0}$ in $S$. Since $I_{\mathfrak{m}} \subset I_{p, \mathfrak{m}}$ evidently, $T_{r_{0}}-\left(1+r_{0}\right) \in I$ shows that $I_{p, \mathfrak{m}}=I_{\mathfrak{m}}$.

Take $S$ to be the set of all primes except $p$ and $q$, and apply Lemma 4 . It follows that $P_{\ell}$ is Eisenstein, i.e., $P_{\ell} \subseteq J_{0}(p)\left[I_{p}\right]_{\ell}^{2} \times J_{0}(q)\left[I_{q}\right]_{\ell}^{2}$. In other words (cf. [6, Chapter II, Corollary 16.4]),

$$
P_{\ell} \subseteq\left(\Sigma(p)_{\ell} \oplus C(p)_{\ell}\right)^{2} \times\left(\Sigma(q)_{\ell} \oplus C(q)_{\ell}\right)^{2} .
$$

Let $\left(\sigma_{1}+c_{1}, \sigma_{2}+c_{2}, \sigma_{3}+c_{3}, \sigma_{4}+c_{4}\right) \in P_{\ell}$ where $\sigma_{1}, \sigma_{2} \in \Sigma(p), \sigma_{3}, \sigma_{4} \in \Sigma(q)$, and $c_{1}, c_{2} \in C(p), c_{3}, c_{4} \in C(q)$. Since $C(p)$ and $C(q)$ consist only of $\mathbb{Q}$-rational torsion points of $J_{0}(p)$ and $J_{0}(q)$, respectively, we have $\left(\sigma_{1}, \sigma_{2}, \sigma_{3}, \sigma_{4}\right) \in P_{\ell}$. For 
any $\tau \in \operatorname{Gal}(\overline{\mathbb{Q}} / \mathbb{Q}),\left(\sigma_{1}^{\tau}, \sigma_{2}^{\tau}, \sigma_{3}^{\tau}, \sigma_{4}^{\tau}\right) \in P_{\ell}$ and $\gamma\left(\sigma_{1}^{\tau}, \sigma_{2}^{\tau}, \sigma_{3}^{\tau}, \sigma_{4}^{\tau}\right)=\gamma\left(\sigma_{1}, \sigma_{2}, \sigma_{3}, \sigma_{4}\right)$. Therefore,

$$
\left(\sigma_{1}^{\tau}-\sigma_{1}, \sigma_{2}^{\tau}-\sigma_{2}, \sigma_{3}^{\tau}-\sigma_{3}, \sigma_{4}^{\tau}-\sigma_{4}\right) \in K_{\ell} \cong \Sigma(p)_{\ell} \times \Sigma(q)_{\ell}
$$

since we are still in the case $\ell \times\left(\frac{p-1}{(p-1,24)}, \frac{q-1}{(q-1,24)}\right)$. It follows that (cf. [3, Theorem 1]) $\sigma_{1}^{\tau}-\sigma_{1}=-\left(\sigma_{2}^{\tau}-\sigma_{2}\right)$ and $\sigma_{3}^{\tau}-\sigma_{3}=-\left(\sigma_{4}^{\tau}-\sigma_{4}\right)$, i.e., $\sigma_{1}+\sigma_{2} \in \Sigma(p)_{\ell}(\mathbb{Q})$ and $\sigma_{3}+\sigma_{4} \in \Sigma(q)_{\ell}(\mathbb{Q})$. Therefore, we obtain $\sigma_{1}=-\sigma_{2}$ and $\sigma_{3}=-\sigma_{4}$, implying that $\left(\sigma_{1}, \sigma_{2}, \sigma_{3}, \sigma_{4}\right) \in K_{\ell}$. This show that $P_{\ell}=K_{\ell} \oplus\left(C(p)_{\ell}^{2} \times C(q)_{\ell}^{2}\right)$. Putting this into (7) shows that $\gamma$ induces an isomorphism

$$
C(p)_{\ell}^{2} \times C(q)_{\ell}^{2} \cong\left(J_{\text {old }}(\mathbb{Q})_{\text {tor }}\right)_{\ell}
$$

This completes the proof of Theorem 2 .

\section{A REMARK ON THE NONTRIVIALity OF CERTAIN EISENSTEIN FACTORS OF $J_{0}(p q)$}

Let $J^{\text {new }} \stackrel{\text { def }}{=} J_{0}(p q) / J_{\text {old }}$ be the new quotient of $J_{0}(p q)$. Let $\mathbb{T}$ be the subring of $\operatorname{End}_{\mathbb{Q}}\left(J^{\text {new }}\right)$ generated over $\mathbb{Z}$ by the Hecke operators. The Eisenstein ideal $I_{p q}$ is the ideal in $\mathbb{T}$ generated by $T_{r}-(1+r)$, for all primes $r \neq p, q$. It is of finite index in $\mathbb{T}$.

Let $\Lambda$ be a non-trivial ideal of $\mathbb{T}$ containing $I_{p q}$ and a prime number $\ell$. Let $a_{\Lambda}=\cap_{n=1}^{\infty} \Lambda^{n}$. The Eisenstein factor $J^{(\Lambda)}$ of $J^{\text {new }}$ associated with $\Lambda$ is defined as

$$
J^{(\Lambda)} \stackrel{\text { def }}{=} J^{\text {new }} / a_{\Lambda} J^{\text {new }} .
$$

In [1], the following theorem is proved:

Theorem 3 (cf. [1, Theorem 4]). Let $p$ and $q$ be distinct prime numbers, $\ell$ an odd prime with $\ell \mid(p+1)$ but $\ell \curlywedge(q-1)$ if $\ell>3$, and $9 \mid(p+1)(q-1)$ but $9 \bigwedge(q-1)$ if $\ell=3$. Then the ideal $\Lambda=\left\langle I_{p q}, \ell, 1-W_{p}\right\rangle$ is a proper ideal of $\mathbb{T}$, and the group $J^{(\Lambda)}(\mathbb{Q})$ is finite.

Remark. Here, $W_{p}$ is induced by the Atkin-Lehner involution on $X_{0}(p q)$, which is derived from the transformation $\tau \mapsto g \tau$ of the Poincaré upper half plane, where $g=\left(\begin{array}{ll}A & B \\ C & D\end{array}\right)$ is any matrix in $M_{2}(\mathbb{Z})$ such that $p|A, p| D, p q \mid C$ and $\operatorname{det}(g)=p$.

A key step in the proof of Theorem 3 in [1] is to produce a point of order $\ell$ in $J^{\text {new }}(\mathbb{Q})$ which is annihilated by $\Lambda$. Given the conditions on $\ell$ in Theorem 3 , by proving that $J_{\text {old }}$ has no $\mathbb{Q}$-rational point of order $\ell$, Berkovič showed that the image of the divisor class of $D^{+,-}=P_{1}+P_{p}-P_{q}-P_{p q}$ in $J^{\text {new }}(\mathbb{Q})$ has order divisible by $\ell$. A suitable multiple of $\overline{D^{+,-}}$would produce the point of $J^{\text {new }}(\mathbb{Q})$ with the desired properties. The constraint $\ell \chi(q-1)$ if $\ell>3$ and $9 X(q-1)$ if $\ell=3$ are necessary for the proof used in [1], because it depends on the following fact: the Galois module $J_{0}(p)(\overline{\mathbb{Q}})_{\ell}$ has a Jordan-Holder factor isomorphic to $\mathbb{Z} / \ell \mathbb{Z}$ or $\mu_{\ell}$ if and only if $\ell \mid \frac{p-1}{(p-1,12)}$. A careful application of Theorems 1 and 2 above allows us to relax some of the constraints on $\ell$ in Theorem 3 .

Theorem 4. Let $p$ and $q$ be distinct prime numbers, $\ell$ an odd prime with $\ell \mid(p+1)$ if $\ell>3$ and $9 \mid(p+1)(q-1)$ and $3 \mid(p+1)$ if $\ell=3$. Then the ideal $\Lambda=\left\langle I_{p q}, \ell, 1-W_{p}\right\rangle$ is a proper ideal of $\mathbb{T}$ and the group $J^{(\Lambda)}(\mathbb{Q})$ is finite. 
The (relaxed) constraints on $\ell$ in Theorem 4 imply that $\ell$ satisfies the conditions in Theorem 2 , so $\left(J_{\text {old }}(\mathbb{Q})_{\text {tor }}\right)_{\ell}$ is known. It is easy to see that $\left(J_{\text {old }}(\mathbb{Q})_{\text {tor }}\right)_{\ell}$ is strictly smaller than $C(p q)_{\ell}$, so an element in its complement in the latter yields an element of $J^{\text {new }}(\mathbb{Q})$ of the desired properties. The details of the proof are left to the reader.

\section{REFERENCES}

1. V. G. Berkovič, The rational points on the Jacobian of modular curves., Math. USSR Sbornik 30 (1976), AMS Translations 478-500.

2. G. Ligozat, Courbes modulaires de genre 1, Bull. Soc. Math. France Mémoire 43 (1975), 5-80. MR 54:5121

3. S. Ling, The old subvariety of $J_{0}(p q)$ and the Eisenstein kernel in Jacobians, Israel J. of Math 84 (1993), 365-384. MR 94h:11047

4. appear in Israel J. of Math.

5. Ju. Manin, Parabolic points and zeta function of modular curves., Izv. Akad. Nauk. SSSR 6 (1972), AMS Translations 19-64. MR 47:3396

6. B. Mazur, Modular curves and the Eisenstein ideal., Pub. Math. I.H.E.S. 47 (1978), 33-186.

7. A. P. Ogg, Rational points on certain elliptic modular curves, Proc. Sym. Pure Math, vol. 20, AMS, 1973, pp. 221-231. MR 49:2743

8. __ Hyperelliptic modular curves, Bull. Soc. Math. France 102 (1974), 449-462. MR 51:514

9. K. Ribet, The old subvariety of $J_{0}(p q)$, Arithmetic Algebraic Geometry, Birkhäuser, 1989, pp. 293-307. MR 92a:11069

10. 100 (1991), 431-476. MR 91g:11066

11.

Department of Mathematics, National University of Singapore, 10 Kent Ridge CresCent, Singapore 119260, Republic of Singapore

E-mail address: matchua@nus.sg

E-mail address: matlings@nus.sg 